\title{
Seroprevalence of hepatitis A infection in a low endemicity country: a systematic review
}

\author{
Ba' Pham* $^{* 1,2}$, Bernard Duval ${ }^{\dagger 3}$, Gaston De Serres ${ }^{\dagger 3}$, Vladimir Gilca ${ }^{\dagger 4}$, \\ Andrea C Tricco ${ }^{\dagger 1}$, Jan Ochnio ${ }^{\dagger 5}$ and David W Scheifele ${ }^{\dagger 5}$
}

\begin{abstract}
Address: ${ }^{1}$ BioMedical Data Sciences, GlaxoSmithKline, Ontario, Canada, ${ }^{2}$ Chalmers Research Group, Children's Hospital of Eastern Ontario Research Institute, Ontario, Canada, ${ }^{3}$ Centre De Recherche du CHUQ, Institut Nationale Sante Publique du Quebec, Quebec, Canada, ${ }^{4}$ Centre de Recherche du CHUQ, Quebec, Canada and ${ }^{5}$ Vaccine Evaluation Centre, University of British Columbia, British Columbia, Canada

Email: Ba' Pham* - ba.z.pham@gsk.com; Bernard Duval - bernard.duval@ssss.gouv.qc.ca; Gaston De Serres - gaston.deserres@ssss.gouv.qc.ca; Vladimir Gilca - vladimir.gilca@ssss.gouv.qc.ca; Andrea C Tricco - andrea.tricco@utoronto.ca; Jan Ochnio - jochnio@interchange.ubc.ca; David W Scheifele - dscheifele@cw.bc.ca

* Corresponding author †Equal contributors
\end{abstract}

Published: 07 July 2005

BMC Infectious Diseases 2005, 5:56 doi:10.1 186/1471-2334-5-56
Received: II May 2005

Accepted: 07 July 2005

This article is available from: http://www.biomedcentral.com/147I-2334/5/56

(C) 2005 Pham et al; licensee BioMed Central Ltd.

This is an Open Access article distributed under the terms of the Creative Commons Attribution License (http://creativecommons.org/licenses/by/2.0), which permits unrestricted use, distribution, and reproduction in any medium, provided the original work is properly cited.

\begin{abstract}
Background: In Canada - a low endemicity country, vaccines for hepatitis A virus (HAV) are currently recommended to individuals at increased risk for infection or its complications. Applying these recommendations is difficult because the epidemiology of HAV infection is poorly defined, complex, and changing. This systematic review aimed to I) estimate age-specific prevalence of HAV antibody in Canada and 2) evaluate infection-associated risk factors.

Methods: MEDLINE (1966-2005) and EMBASE (1980-2005) were searched to identify relevant studies for the systematic review. Archives for the Canada Diseases Weekly Report (1975-1991) and Canada Communicable Disease Report (1992-2005) were searched for relevant public health reports. Data were abstracted for study and participants' characteristics, age-specific prevalence, and risk factors.
\end{abstract}

Results: A total of 36 reports describing 34 unique studies were included.

The seroprevalence in Canadian-born children was approximately $1 \%$ in ages $8-13,1-6 \%$ in $20-24,10 \%$ in $25-29,17 \%$ in 30-39, and increased subsequently. In age groups below 20 and 20-29, age-specific seroprevalence generally remained constant for studies conducted across geographic areas and over time.

Compared to Canadian-born individuals, subjects born outside Canada were approximately 6 times more likely to be seropositive (relative risk: 5.7 [95\% Cl 3.6, 9.0]). Travel to high risk areas in individuals aged 20-39 was associated with a significant increase in anti-HAV seropositivity (RR $2.8[1.4,5.5]$ ). Compared to heterosexuals, men having sex with men were only at a marginally higher risk (adjusted odds ratio $2.4[0.9,6.1]$ ). High risk for seropositivity was also observed for Canadian First Nations and Inuit populations.

Conclusion: Results from the current systematic review show that in this low endemicity country, disease acquisition occurs in adulthood rather than childhood. The burden of disease is high; approximately $\mathrm{I}$ in 10 Canadians had been infected by ages 24-29. The increase in prevalence in young adults coincides with disease importation and increasing frequency of risk factors, most likely behavioral-related ones.

Gaps in seroprevalence data were identified rendering the application of current immunization recommendations difficult. A nationwide prevalence survey for all Canadians is needed. This is essential to quantify the effectiveness of current recommendations and conduct cost-effectiveness evaluations of alternative immunization programs, if necessary. 


\section{Background}

Hepatitis A virus (HAV) is prominent in many areas of the world [1-3]. In North America, infection rates have declined with better hygiene practice and public sanitation but remain heterogeneous across geographic and socioeconomic strata [4-6]. Further decline is possible with HAV vaccines which provide consistent, long-lasting protection and have been available since the mid-1990s $[7,8]$. In the United States, universal vaccination of children and youth has been in place for about 6 years in high endemicity areas [9], leading to historically low rates nationally in recent years [10]. In Canada, the current national immunization guide recommends HAV vaccines for individuals at increased risk of infection or its complications. The guide also states that a universal immunization program should be considered, but further discussion is needed nationally [11].

Applying the Canadian recommendations is difficult because the epidemiology of HAV infection in Canada is poorly defined, complex, and changing [8]. Reported rates differ substantially by province, gender, and age [12]. The rates show repeated peaks and troughs [13] and the last peak occurred in mid-1990 [12,13]. It was during the subsequent period of decline that vaccines were used as a tool to enhance HAV control [8].

Evaluating the impact of the current recommendations is also difficult. Data are needed to distinguish between a cyclical decline and a further decline associated with the recommendations [10]. Such assessment is important to inform future immunization policies. A combination of timely case-notification data, prevalence data, and risk factor data is required for both the application and evaluation of the recommendations.

Case-notification data is of limited use due to underdetection of sub-clinical infection and under-reporting of confirmed cases $[8,14]$. A useful means that circumvent these limitations is to measure the prevalence of HAV antibody [15]. Following an acute infection, antibody to HAV develops in virtually every instance, remaining detectable for decades, and providing a reliable marker of past infection. In the United States, countrywide seroprevalence surveys and sentinel surveillance have been conducted to provide insight into HA epidemiology, and to rationalize and evaluate immunization programs $[10,15,16]$. In Canada, similar surveys exist but are limited in scope and comprehensiveness [17-19]. Consequently, risk factor data are also limited and fragmented $[12,17]$. The current systematic review aimed to 1 ) estimate age-specific prevalence of hepatitis A antibody in Canada and 2) evaluate infection-associated risk factors.

\section{Methods}

MEDLINE (Jan. 1966 - Mar. 2005) and EMBASE (Jan.1980 - Mar. 2005) were searched to identify citations of potentially relevant studies for the systematic review (MeSH terms: "hepatitis" exploded AND "Canada" exploded). A study report was included if it contained prevalence data of HAV-antibody (detected through sera or saliva samples, hereafter referred to as seroprevalence) for a Canadian population. Reasons for exclusion were categorized and reported. Citations were screened independently by two reviewers. Full-text study reports from citations deemed relevant by one reviewer were obtained. Archives for the Canada Diseases Weekly Report (Jan. 1975 - Dec. 1991, the last year of reporting) and Canada Communicable Disease Report (Jan. 1992 - Mar. 2005) were also searched for potentially relevant public health reports [20]. Two reviewers independently reviewed both published and public health reports. Discrepancies were resolved through discussion. Back referencing and author searches of all included studies were conducted. Other potentially relevant reports were obtained by contacting HA experts and related public health units.

From the included reports, data were independently abstracted for study and participants' characteristics. Agespecific prevalence of HAV antibody data were extracted for Canadian-born participants, all Canadians including individuals born outside the country, and participants with known risk factors [11]. Seroprevalence estimates and 95\% confidence intervals (CI) were derived assuming a binomial distribution for the number of seropositive individuals from the total numbers of tested individuals. Participants who reported receiving the HA vaccine were excluded from the prevalence estimates as they were likely to have vaccine-induced antibody.

If available, adjusted odds ratios (AOR) for seropositivity of both demographics and risk factors were extracted, together with the baseline risk of HAV seropositivity (i.e., population, location and timing of the survey). When the AOR of a variable was not reported, HAV antibody data stratified by the variable were obtained to derive the unadjusted relative risk for seropositivity (URR). Relative risk was used as it is a better risk estimate than the odds-ratio in the range of seroprevalence observed in this systematic review [21]. If appropriate, a random effects model was used to combine URRs across studies, together with an assessment for heterogeneity (i.e., chi-squared test).

Information related to the risk of seropositivity was summarized for the following risk categories [11]: 1) travelers to high endemicity areas; 2 ) groups with high risk activities such as men who have sex with men (MSM), illicit drug users, and street people; 3) First Nations and Inuit populations; and 4) others (e.g., individuals with chronic 
Table I: Study and participant characteristics

\begin{tabular}{|c|c|c|c|c|c|c|}
\hline Study & $\begin{array}{l}\text { Study } \\
\text { Design }\end{array}$ & Study Year & $\mathbf{n}$ & Population & Location & Age in years \\
\hline $\begin{array}{l}\text { PUBLISHED } \\
\text { LITERATURE }\end{array}$ & & & & & & $\begin{array}{l}\text { Mean } \pm \text { SD or } \\
\text { (range) }\end{array}$ \\
\hline Ochnio 2005* [22] & $P$ & $2000-1$ & 811 & Grade 9 students & British Columbia & $(14-15)$ \\
\hline Muecke 2004* [23] & $\mathrm{CC}$ & 2001 & 492 & Day-care educators & Montreal & 37 \\
\hline Minuk 2003 [24] & $P$ & 1999 & 315 & First Nations & Manitoba & $34 \pm 15$ \\
\hline Ochnio 200I [25] & $P$ & 1998 & 494 & Street youth, IDU, MSM & Vancouver & $19,35,34$ \\
\hline Smieja 200I [26] & $\mathrm{CC}$ & 1997-8 & 179 & IHD patients & Hamilton & $6 I(38-8 I)$ \\
\hline Kiefer 2000 [27] & $\mathrm{R}$ & 1997 & 343 & Hepatitis C patients & Edmonton & $40(0-95)$ \\
\hline Allard 200I [28] & $P$ & 1995-97 & 353 & Gay men & Montreal & 37 \\
\hline Moses 2002 [29] & $P$ & $1995-6$ & 533 & Street people & Winnipeg & $26(11-65)$ \\
\hline Roy 2002 [30] & $\mathrm{R}$ & $1995-6$ & 427 & Street youth & Montreal & $(14-25)$ \\
\hline Ochnio 1997 [31] & $P$ & $1995-6$ & 224 & Grade 6 students & Vancouver & $(10-12)$ \\
\hline De Serres 1997 [32] & $P$ & 1995 & 85 & Sewer workers & Quebec & $36 \pm 7$ \\
\hline De Serres 1995 [33] & $\mathrm{CC}$ & 1995 & 228 & Sewer workers & Quebec & $41(28-64)$ \\
\hline Smieja 2003 [34] & $\mathrm{R}$ & $1993-5$ & 3127 & $\mathrm{CV}$ or high risk diabetes & Canada & 65 \\
\hline Payment I99| [18] & $P$ & $1988-9$ & 617 & French-Canadian & Montreal & $(9-79)$ \\
\hline Embil 1989 [35] & $P$ & $|98|-3$ & $\begin{array}{l}2036 \\
1922\end{array}$ & $\begin{array}{l}\mathrm{I} / \mathrm{CF} \text { recruits } \\
2 / \mathrm{CF} \text { males }\end{array}$ & $\begin{array}{l}\text { Nova Scotia, } \\
\text { Quebec, Posted } \\
\text { abroad }\end{array}$ & $\begin{array}{l}1 /(15-25) \\
2 / 26(17-53)\end{array}$ \\
\hline $\begin{array}{l}\text { Nicolle I986 [36], Minuk } \\
\text { I985 [37] }\end{array}$ & $P$ & 1982 & 172 & Chesterfield Inlet & $\begin{array}{l}\text { Northwest } \\
\text { Territories }\end{array}$ & $0-78$ \\
\hline Crewe $1983[38]$ & $\mathrm{P}$ & $|98|-2$ & 304 & Children attending outpatient clinic & Halifax & $(0.5-16)$ \\
\hline $\begin{array}{l}\text { Minuk } 1982[39] \\
\text { Minuk } 1982[40]\end{array}$ & $P$ & 1980 & 720 & Inuit & $\begin{array}{l}\text { Northwest } \\
\text { Territories }\end{array}$ & $(0.3-86)$ \\
\hline McFarlane $1980[4 I]$ & $P, R$ & 1980 & $\begin{array}{l}243 \\
152 \\
293 \& 282\end{array}$ & $\begin{array}{l}\text { I/STD clinic patients } \\
2 / \text { Student nurses } \\
3 / 2 \text { groups of blood donors }\end{array}$ & Nova Scotia & $\begin{array}{l}1 /(16-26) \\
2 /(18-24) \\
3 /(16-26) \& \\
(5 I-65)\end{array}$ \\
\hline Buchner 1980 [42] & $\mathrm{R}$ & 1980 & 5097 & Blood donors & Toronto & $<21,>60$ \\
\hline Richer 1982 [55] & $\mathrm{R}$ & $1970-79$ & 447 & Samples of acute viral hepatitis & Montreal & Not reported \\
\hline Minuk 1994 [43] & $P$ & $1974-8$ & 42 & Household transmission & Winnipeg & $27 \pm 12$ \\
\hline Minuk 2003 [44] & SR & $1980-2000$ & 1706 & Inuit and First Nations & Various locations & $0-60+$ \\
\hline McFarlane 1982 [45] & $P$ & NR & 154 & Institutions & Nova Scotia & $(13-28)$ \\
\hline McFarlane 198I [46] & $P$ & NR & 130 & $\begin{array}{l}\text { Patients with hematological } \\
\text { malignancy }\end{array}$ & Nova Scotia & $(4-76)$ \\
\hline
\end{tabular}

\section{GREY LITERATURE}

\begin{tabular}{|c|c|c|c|c|c|c|}
\hline Duval 2005* [48] & $P$ & 2003 & 1057 & Canadian aged 8-13 & Canada & $(8-13)$ \\
\hline Wu 2005 [47] & $\mathrm{R}$ & 1992-9 & NR & Subjects tested for HAV infection & Manitoba & Not reported \\
\hline Ochnio 2004 [49] & $P$ & 2003 & 585 & Young adults & Vancouver & $(20-39)$ \\
\hline Cook 2000 [19] & $\mathrm{R}$ & 2000 & 1206 & Women of child-bearing age & British Columbia & $(15-44)$ \\
\hline Harb $2000[50]$ & $P$ & 1999 & 172 & First Nations & British Columbia & $(0-40+)$ \\
\hline Levy 2001 [5I] & $P$ & 1997 & 1000 & University students & Toronto & $25 \pm 5$ \\
\hline Ford-Jones 1995 [52] & $\mathrm{P}$ & 1993 & 122 & Day-care providers & Toronto & Not reported \\
\hline Ochnio 1995 [53] & $\mathrm{P}$ & $1994-5$ & 1019 & Clients to travel clinic & Vancouver & $(2-69+)$ \\
\hline Kocuipchyk I995 [54] & $P$ & $1991-2$ & 505 & Individuals attending travel clinic & Edmonton & $(16-60+)$ \\
\hline
\end{tabular}

Notes:*Study reported seroprevalence data for individuals with or without HAV vaccination.

Abbreviations: Study design: P prospective data acquisition, R retrospective data acquisition, CC case control, SR systematic review. Population: IDU injection drug users, MSM men who have sex with men, IHD ischemic heart disease, CV cardiovascular, CF Canadian Forces, STD sexually transmitted disease. 
hepatitis, household contacts, infected food handlers, etc.).

\section{Results \\ Literature search}

A total of 36 reports describing 34 unique studies were included in the systematic review (Table 1) [18,19,22-55]. These were obtained from screening 413 potentially relevant citations and reviewing 66 full-text study reports and 25 public health reports (Figure 1). Common reasons for exclusion at the screening stage included studies of hepatitis B virus $(n=95)$, hepatitis C virus $(n=64)$, commentaries $(\mathrm{n}=24)$, and others $(\mathrm{n}=121$; Figure 1$)$. Common reasons for exclusion at the full-text review stage included general review of HAV $(n=9)$, no seroprevalence data ( $n$ $=17)$, and other viral hepatitis $(\mathrm{n}=16$; Figure 1$)$.

Overall, $74 \%(n=25)$ of the included studies were reported in peer-reviewed journals while $26 \%(n=9)$ were grey literature [20], including $9 \%(\mathrm{n}=3)$ public health reports, $14 \%(\mathrm{n}=5)$ abstracts, and $3 \%(\mathrm{n}=1)$ unpublished study (Table 2). HAV antibody was detected using serum samples in 28 studies and saliva samples in 6 . The median sample size was 427 and 793 for the published and grey literature studies, respectively. Only $21 \%$ (7/34) of all studies reported prevalence among Canadian-born participants and 29\% (10/34) reported prevalence data of all participants including foreign-born individuals. The majority of these studies (27/34) reported prevalence data of participants with known risk factors.

\section{Age-specific seroprevalence}

The seroprevalence in Canadian-born children aged 8-13 was $1 \%$ [95\% CI: $0.5-2 \%$ ] according to a national survey conducted in 2003 [48]. The seroprevalence was $1-6 \%$ in ages $20-24$, approximately $10 \%$ in $25-29,17 \%$ in $30-39$, and increased subsequently (Figure 2). In age groups below 20 and 20-29, age-specific seroprevalence generally remained constant for studies conducted across geographic areas in 1980, 1988, 1997, and 2003. This remained so despite differences in study methodology.

There was no association between seropositivity and gender based on 9 population comparisons from 5 studies (n $=4158$, URR: 1.0 [95\% CI: 0.9, 1.1]) [22,31,35,41,54], which was consistent with results of 3 other studies reporting adjusted risk estimates (Table 3) [25,34,48]. Two studies in the early 1980's suggested that individuals living in urban areas were 30\% more likely to have HAV antibody compared to those in rural areas $(n=647$, URR: $1.3[1.2,1.5])[41,46]$.

Compared to Canadian-born individuals, subjects born outside Canada were approximately 6 times more likely to

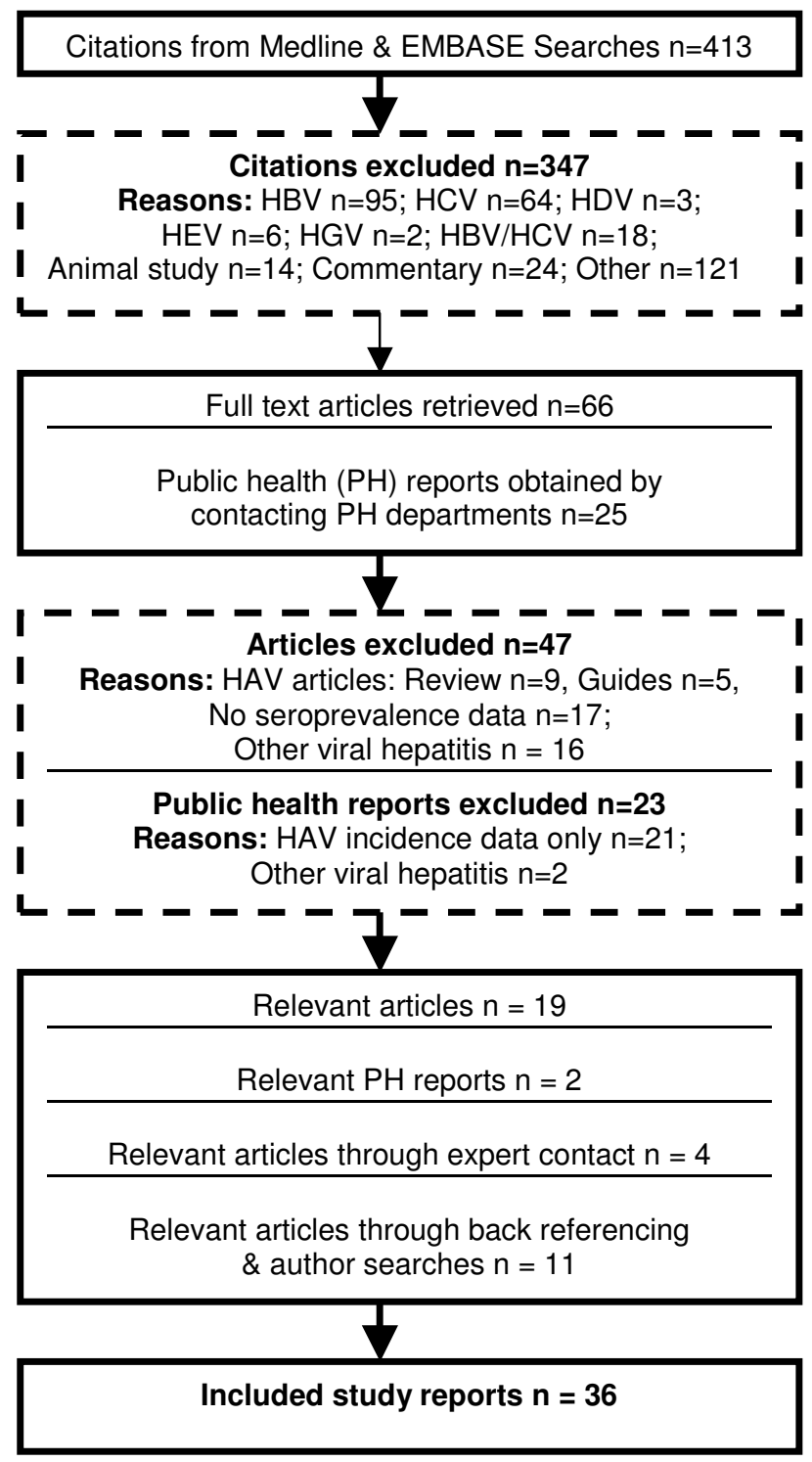

Figure I

Results of the literature search.

be seropositive ( $\mathrm{n}=3008$, URR: 5.7 [3.6, 9.0], Table 3) $[30,31,49,51,53]$, which most likely occurred in their birth country. However, the possibility that infection occurred in Canada could not be ruled out. Age-specific seroprevalence estimates including these individuals varied substantially and could only be used to infer the level of immunity in the population. For example, seroprevalence among all Canadians aged $<20$ ranged from $2-16 \%$ $[18,31,38,48,51]$ for which $3-25 \%$ of the sampled populations were individuals born outside the country 
Table 2: Study characteristics

\begin{tabular}{|c|c|c|}
\hline & Published Literature $(n=25)$ & Grey Literature $(n=9)$ \\
\hline & Peer-reviewed study report $(n=25)$ & $\begin{array}{l}\text { Public health report }(n=3) \\
\text { Abstract }(n=5) \\
\text { Unpublished report }(n=1)\end{array}$ \\
\hline \multicolumn{3}{|l|}{ Study Design } \\
\hline Case-control & 2 & 0 \\
\hline Prospective $(\mathrm{P})$ data acquisition & 16 & 7 \\
\hline Retrospective (R) data acquisition & 5 & 2 \\
\hline P \& R data acquisition & I & 0 \\
\hline Systematic review & $\mathrm{I}$ & 0 \\
\hline \multicolumn{3}{|l|}{ Sample Size } \\
\hline$>1000$ & 4 & 3 \\
\hline $100-1000$ & 19 & 6 \\
\hline$<100$ & 2 & 0 \\
\hline Median [I st, $3^{\text {rd }}$ Quartile] & $427[224,720]$ & $793[422,1029]$ \\
\hline Mean (Min, Max) & $877(42,5097)$ & $708(122,1206)$ \\
\hline \multicolumn{3}{|l|}{ Timing of data collection } \\
\hline $2000-2004$ & 2 & 3 \\
\hline $1990-1999$ & 12 & 6 \\
\hline $1980-1989$ & 7 & 0 \\
\hline $1970-1979$ & 2 & 0 \\
\hline Not reported & 2 & 0 \\
\hline \multicolumn{3}{|l|}{ Populations with prevalence data } \\
\hline Canadian born subjects & 4 & 3 \\
\hline All Canadians $\mathbb{1}$ & 6 & 4 \\
\hline Participants with known risk factors & 21 & 7 \\
\hline \multicolumn{3}{|l|}{ Seropositivity test } \\
\hline Serum samples & 22 & 6 \\
\hline Saliva samples & 3 & 3 \\
\hline
\end{tabular}

Notes: Tincluding subjects born outside of Canada

$[25,48,53]$. This contrasted the $1 \%$ seropositivity for Canadian-born participants reported above.

\section{Risk factors}

Travel-related data were available in 6 studies (Table 1) $[23,35,48,49,53,54]$. HAV antibody prevalence for Canadian-born individuals visiting a travel clinic was $2.3 \%$ in ages $20-25$ and $4.3 \%$ in ages $25-28$ [53]. The prevalence of seropositivity in these individuals was comparable to that reported above for Canadian-born individuals. The risk related to travel among Canadian-born was also not significant in a study of day-care educators [23]. Two population-based surveys reported travel-related risk $[48,49]$. In one study, travel to high risk areas by Canadian-born individuals aged 20-39 (approximately 12\% of study participants) was associated with a significant increase in seropositivity $(\mathrm{n}=407$, URR: $2.8[1.4,5.5])$ [49]. In a national survey of children aged $8-13$, the prev- alence was $1.9 \%$ in Canadian-born non-vaccinated travelers and $1.3 \%$ in non-travelers; the association was again not significant (Table 3) [48].

Two studies evaluated HAV infection among MSM in two different cities $[25,28]$. MSM participants on average had 3 sexual partners over the preceding 6 months, according to one study [28]. Also, $18 \%$ of these individuals were food handlers. Compared to heterosexuals, MSM were only at a marginally higher risk for seropositivity $(\mathrm{n}=494$, AOR $2.4[0.9,6.1])$, according to the second study [25]. However, the study sample was highly heterogeneous and included MSM, injection drug users (IDU), and street youth.

Data on street-involved populations were available in three studies (Table 3) $[25,29,30]$. Seropositivity was approximately 5\% in street youth aged 14-25 in Vancou- 


Author
Crewe 1983
Payment 1991
Dunal 2004
Lewy 2001
Ochnio 1997
Crewe 1983
Ochnio 2004
Lewy 2001
Ochnio 2004
Payment 1991
Lewy 2001
MucFarlane, 1980
Ochnio 2004
Lewy 2001
Ochnio 2004
Payment 1991
Payment 1991
Payment 1991
Mayment 1991

Population

Age $<20$

Children, Halitax, 1981-2

French-Canadian, Montreal, 1988-9

Canadian children, Canada, 2003

University students, Toro nto, 1997

G6 students, Vancouver, 1995-6

Children, Haligx, 1981-2

Age $20 \cdot 29$

Young adults, Vancouver, 2003

University students, Toro nto 1997

Young adults, Vancouver, 2003

French-Canadian, Montreal, 1988-9

University students, Toro nto, 1997

Blood donors, nural NS, 1980

Age $30-39$

Young adults, Vancouver, 2003

Universty students, Toro nto, 1997

Young adults, Vancouver, 2003

French-Canadian, Montreal, 1988-9

Age $40-49$

French-Canadian, Montreal, 1988-9

40.49

Age $>50$

Blood donors, nural NS, 1980

$51.65 \quad 167$

French-Canadian, Montteal, 1988-9 50-59 81

French-Canadian, Montreal, 1988-9 60-79 89 $\begin{array}{lllllllllll}0 & 10 & 20 & 30 & 40 & 50 & 60 & 70 & 80 & 90 & 100\end{array}$

$\begin{array}{ll}\text { Age } & n \\ 0-9 & 209 \\ 9-19 & 159 \\ 8-13 & 969 \\ 18-19 & 66 \\ 10-12 & 167 \\ 10-16 & 95 \\ & \\ & \\ 20-24 & 86 \\ 20-24 & 370 \\ 25-29 & 96 \\ 20-29 & 87 \\ 25-29 & 183 \\ 16-26 & 140\end{array}$

30-34 117 30-34 79 $35-39 \quad 110$

$30-39 \quad 94$
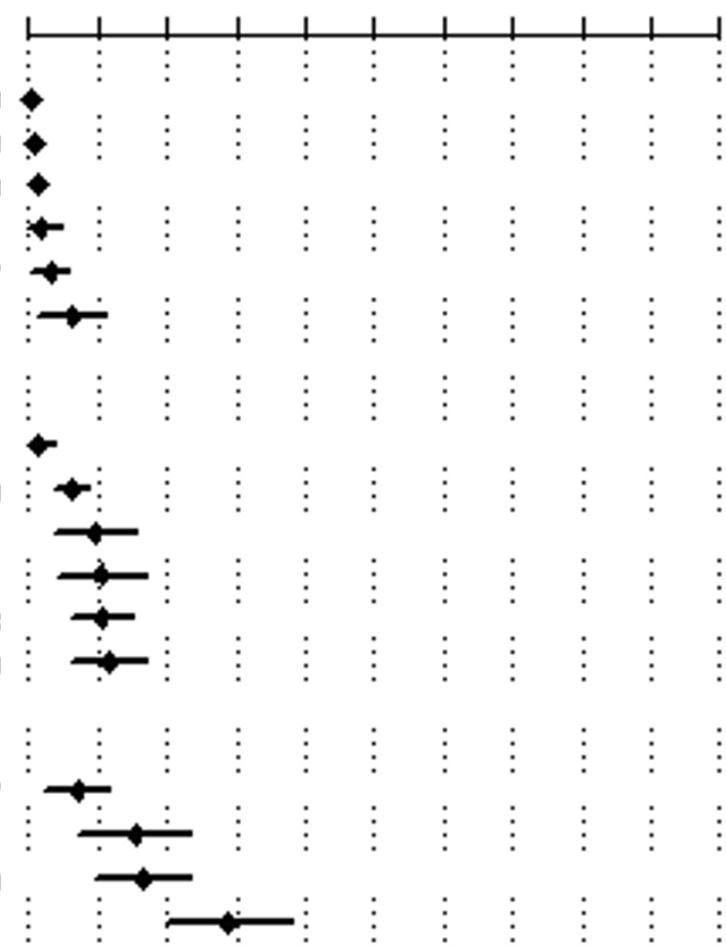

$\div \quad: \quad: \quad: \quad:$

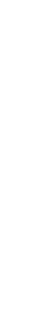


Table 3: Assessment of risk factors

\begin{tabular}{|c|c|c|c|c|c|c|}
\hline Risk factor & $\mathbf{n}$ & $\begin{array}{l}\text { Risk } \\
\text { Measure }\end{array}$ & $\begin{array}{l}\text { Risk } \\
\text { Estimate } \\
(95 \% \mathrm{Cl})\end{array}$ & $\begin{array}{l}\text { Population, Location, Timing } \\
\text { of Data Acquisition }\end{array}$ & $\begin{array}{l}\text { Age in } \\
\text { years }\end{array}$ & Study \\
\hline DEMOGRAPHICS & & & & & $\begin{array}{l}\text { Mean } \pm \text { SD } \\
\text { or (range) }\end{array}$ & \\
\hline Female versus Male & 1003 & AOR & $2.2(0.8,6.25)$ & $\begin{array}{l}\text { School-aged children, Canada, } \\
2003\end{array}$ & $(8-13)$ & {$[48]$} \\
\hline Female versus Male & 3128 & AOR & $0.8(0.6,0.96)$ & $\begin{array}{l}\text { CV or high risk diabetes, Canada, } \\
\text { 1993-5 }\end{array}$ & 65 & [34] \\
\hline Female versus Male & 494 & AOR & $1.3(0.8,2.3)$ & SY, MSM, IDU, Vancouver, I998 & $32 \pm 11$ & {$[25]$} \\
\hline Female versus Male & 4158 & URR & $\begin{array}{l}1.0(0.9 .1 .1) \\
{[p=0.30]^{*}}\end{array}$ & $\begin{array}{l}9 \text { population comparisons } \\
\text { from } 5 \text { studies }\end{array}$ & $(8-65+)$ & {$[31,35,41,49,54]$} \\
\hline Urban versus Rural & 647 & URR & $\begin{array}{l}1.3(1.2,1.5) \\
{[p=0.59]^{*}}\end{array}$ & $\begin{array}{l}3 \text { population comparisons } \\
\text { from } 2 \text { studies }\end{array}$ & $(16-76)$ & {$[41,46]$} \\
\hline $\begin{array}{l}\text { Born in high risk country versus } \\
\text { born in Canada }\end{array}$ & 494 & AOR & $2.9(1.1,7.6)$ & SY, MSM, IDU, Vancouver, 1998 & $32 \pm 11$ & {$[25]$} \\
\hline $\begin{array}{l}\text { Born in endemic country versus } \\
\text { born in Canada }\end{array}$ & 1003 & AOR & $22.3(6.6,75.0)$ & $\begin{array}{l}\text { School-aged children, Canada, } \\
2003\end{array}$ & $(8-13)$ & [48] \\
\hline Foreign-born versus Canadian-born & 353 & AOR & $6.2(2.6,15.0)$ & Gay men, Montreal, 1995-97 & 36 & [28] \\
\hline $\begin{array}{l}\text { Born in a high-income country } \\
\text { versus moderate to low } \dagger\end{array}$ & 492 & AOR & $20.8(9.4,46.0)$ & $\begin{array}{l}\text { Day-care educators, Montreal, } \\
200 \mathrm{I}\end{array}$ & 37 & {$[23]$} \\
\hline $\begin{array}{l}\text { Foreign-born versus Canadian- } \\
\text { born }\end{array}$ & 3008 & URR & $\begin{array}{l}5.7(3.6,9.0) \\
{[p<0.01]^{*}}\end{array}$ & $\begin{array}{l}5 \text { population comparisons } \\
\text { from } 5 \text { studies }\end{array}$ & $(2-69+)$ & {$[30,31,49,51,53]$} \\
\hline
\end{tabular}

\section{TRAVEL TO HIGH RISK}

AREA

\begin{tabular}{|c|c|c|c|c|c|c|}
\hline $\begin{array}{l}\text { Travel to high risk area versus } \\
\text { otherwise }\end{array}$ & 1003 & AOR & I.4 $(0.4,4.8)$ & $\begin{array}{l}\text { School-aged children, Canada, } \\
2003\end{array}$ & $(8-13)$ & [48] \\
\hline $\begin{array}{l}\text { Travel to high risk areas versus } \\
\text { otherwise }\end{array}$ & 407 & URR & $2.8(1.4,5.5)$ & $\begin{array}{l}\text { Canadian-born adults, Vancouver, } \\
2003\end{array}$ & $(20-39)$ & [49] \\
\hline $\begin{array}{l}\text { Ever travelled to a developing } \\
\text { country } \ddagger\end{array}$ & 492 & AOR & $2.4(1.3,4.2)$ & $\begin{array}{l}\text { Day-care educators, Montreal, } \\
2001\end{array}$ & 37 & [23] \\
\hline
\end{tabular}

\section{HIGH RISK ACTIVITIES}

\begin{tabular}{|c|c|c|c|c|c|c|}
\hline MSM versus heterosexuals & 494 & AOR & $2.4(0.9,6.1)$ & SY, MSM, IDU, Vancouver, 1998 & $(25-34)$ & [25] \\
\hline $\begin{array}{l}\text { Sexual partners with VH history } \\
\text { versus otherwise }\end{array}$ & 420 & AOR & $13.8(4.2,45.2)$ & Street youths, Montreal, 1995-6 & $(14-25)$ & [30] \\
\hline $\begin{array}{l}\text { Insertive anal penetration versus } \\
\text { otherwise }\end{array}$ & 420 & AOR & $5.1(1.6,16.7)$ & Street youths, Montreal, 1995-6 & $(14-25)$ & [30] \\
\hline History of STD versus no history & 500 & AOR & $2.0(1.2,3.3)$ & Street people, Winnipeg, 1995-6 & $26(11-65)$ & [29] \\
\hline History of IDU versus no history & 494 & AOR & $6.5(1.6,26.3)$ & SY, MSM, IDU, Vancouver, 1998 & $(25-34)$ & [25] \\
\hline History of IDU versus no history & 500 & AOR & $1.6(0.99,2.7)$ & Street people, Winnipeg, 1995-6 & $26(11-65)$ & [29] \\
\hline
\end{tabular}

\section{FIRST NATIONS AND INUIT}

\begin{tabular}{|c|c|c|c|c|c|c|}
\hline Native versus Non-native & 1003 & AOR & $5.2(1.0,26.0)$ & $\begin{array}{l}\text { School-aged children, Canada, } \\
2003\end{array}$ & $(8-13)$ & [48] \\
\hline Aboriginal versus Non-aboriginal & 500 & AOR & $6.6(3.8,11.5)$ & Street people, Winnipeg, 1995-6 & $26(11-65)$ & [29] \\
\hline Inuit versus white in NWT & 708 & URR & $4.5(2.4,8.5)$ & Inuits, Baker Lake, NWT, 1980 & $(0.3-86)$ & {$[39,40]$} \\
\hline $4+$ versus I-3 household occupants & 635 & URR & I.I $(0.98,1.3)$ & Canadian Inuit, Baker Lake, 1980 & $(0.3-86)$ & {$[39,40]$} \\
\hline
\end{tabular}

\section{OTHERS}

Years working in day-care, 5-year groups§

History of daycare versus no history

\section{AOR}

1278 URR
I.3 (I.0, I.8)

$1.2[0.7,2.2]$ $[\mathrm{p}=0.30]^{*}$
Canadian-born day-care educators, Montreal, $200 \mathrm{I}$

2 population comparisons

from 2 studies

$\begin{array}{ll}34 & {[23]} \\ 8-13 & {[31,48]}\end{array}$

8-13

$[31,48]$ 
Table 3: Assessment of risk factors (Continued)

\begin{tabular}{|c|c|c|c|c|c|c|}
\hline Sewer workers versus controls|| & 228 & URR & T.I $(0.8,1.4)$ & $\begin{array}{l}\text { Sewer workers, Quebec City, } \\
1993\end{array}$ & $4 \mid(28-64)$ & [33] \\
\hline $3+$ versus $0-3$ siblings & 502 & URR & $2.0(1.7,2.5)$ & Travel clinic, Edmonton, 1991-2 & $(16-60+)$ & [54] \\
\hline $\begin{array}{l}\text { Current household income } \\
<20,000 / y r \|\end{array}$ & 153 & AOR & $5.3(1.2,24.2)$ & $\begin{array}{l}\text { Foreign-born day-care educators, } \\
\text { Montreal, 200I }\end{array}$ & 39.7 & [23] \\
\hline $\begin{array}{l}\text { Annual family income }<30,000 \text { vs } \\
\geq 30,000\end{array}$ & 1057 & URR & $0.7(0.3,2.0)$ & $\begin{array}{l}\text { School-aged children, Canada, } \\
2003\end{array}$ & $(8-13)$ & [48] \\
\hline
\end{tabular}

Abbreviations: AOR adjusted odds ratio. CV cardiovascular. SY street youth. MSM men who have sex with men. IDU injectable drug user. URR unadjusted relative risk. STD sexually transmitted disease. NWT North West Territory. G6 grade 6. yr year

Notes: *Meta-analytical estimates $(95 \% \mathrm{Cl})$ [p-value from a test of homogeneity] from random-effects models. $\dagger$ The day-care educator study included 492 participants, including 339 Canadian-born individuals and 153 foreign-born. The odds-ratio for "born in a high-income country versus moderate to low" was 20.8 (95\% Cl 9.4, 46.0) for all 492 participants, not reported for Canadian-born, and 4.6 (I.7, I2.2) for foreign-born. $\ddagger$ The odds-ratio for "ever traveled to a developing country" in the day-care educators study was 2.4 (I.3, 4.2$)$ for all 492 participants, not significant for Canadian-born (estimated OR not available), and 8.I (2.3, 29.0) for foreign-born. $§$ The odds-ratio for "years working in day-care, 5-year groups" was not significant for all 492 participants (estimated OR not available), I.3 (I.0, I.8) for Canadian-born, and not significant (estimated OR not available) for foreign-born. || Control subjects were outpatients undergoing lipid testing,. ๆ The odds-ratio for "current household income <20,000/ $\mathrm{yr}^{\prime \prime}$ in the day-care educators study was not significant (estimated OR not available) for all 492 participants and Canadian-born and was 5.3 (I.2, 24.2) for foreign-born.

not associated with seropositivity [31]. Also, the seropositivity was 1.3-1.6 times higher in children aged 8-13 who attended day-care, but no statistical difference was evident in all participants, non-vaccinated participants, or those without known risk factors [48].

Other potential risk factors were also examined. From a sample of 343 individuals who tested positive for hepatitis C, 30\% of those aged 20-29 were seropositive for HAV [27]. One prospective cohort followed 62 household contacts and 20 index cases over 6 months; the risk of infection was $52 \%$ among other susceptible household members [43]. Working in a sewage plant was not associated with seropositivity [33].

\section{Discussion}

The seroprevalence data consolidated in this systematic review had many limitations. Except for one national survey in ages 8-13 [48], other studies were generally not representative of the general population. Substantial variation across studies was observed with respect to study population, timing, sample size, and location. Reporting of data was inconsistent with respect to age stratification and definition of risk factors. Some studies conducted after the introduction of the vaccine around 1997 did not take vaccine-induced HAV antibody into account. This was, however, rectified in more recent studies $[23,48,49]$. For example, the seroprevalence in a national survey of children aged $8-13$ was $2.7 \%$ overall and $2.0 \%$ after the exclusion of self-reported vaccinees [48]. The corresponding figures in a survey of young adults aged 20-39 were $22 \%$ and $16 \%$, respectively [49]. Given these limitations, improvements in the reporting of future HAV prevalence studies are required. Most importantly, prevalence data should be stratified by participants' birthplace and account for vaccine-induced antibody.

Results from the current systematic review show that disease acquisition occurs in adulthood rather than childhood [14]. In Canada, the increase in prevalence in young adults coincides with disease importation and increasing frequency of behavioral risk factors, such as risk activities among MSM and street-involved populations. Even in this low endemicity country, approximately 1 in 10 Canadians had been infected by ages 24-29.

A low level of HAV immunity in Canada is evident from this systematic review. Over $90 \%$ of Canadian-born individuals aged 20-29, and over $80 \%$ of those aged $30-39$ remained unprotected. Canadians born outside the country generally have a higher prevalence of HAV antibody, yet including these individuals did not significantly improve the percentage of protected Canadians. This low level of immunity and persistent risk of exposure to HAV suggest that outbreaks are possible in the future $[14,47]$. For example, unprotected clients exposed to an infected food handler led to mass immunizations in the early 2000's (Toronto 2002 [56], n = 19,208; London 2002 [57], $\mathrm{n}=16,320$; Vancouver 2002 [58] $\mathrm{n}=6,000)$.

Clarifications are required to better understand the epidemiology of HAV in Canada, especially the inter-relation between timely case-notification data, seroprevalence data, and risk factor data. In a study examining national case-notification data from 1990-1999, estimated incidence of reported cases decreased while the average age of exposure and subsequent infection increased [12]. Given the low seroprevalence in Canadian youth, the current 
results suggest that the average age of HA exposure is above 24 and is increasing. While infection in children is often sub-clinical or mild, infected adults often experience more severe symptoms $[1,59]$.

Results of the current systematic review are consistent with low HAV endemicity patterns in developed countries [60-62]. A substantial burden of infection was observed in young Canadians and this did not decrease among successive generations over the past 20 years. Similar observations were reported elsewhere [63]. In these low endemicity countries, outbreaks are common [64,65]. Sources of outbreaks that are common in these countries include infected food handlers [56,57], contaminated food importation $[66,67]$, and unprotected immigrants who visit friends and relatives in their original countries [68].

In order to apply the current immunization recommendations, substantial information pertaining to groups at increased risk of HA infection or its complications is required. Results from this systematic review suggest that the risk of HA infection in these target groups was not well documented. With the exception of a few populationbased surveys $[48,49]$, most studies enrolled participants with known risk factors and failed to include a control group. In addition, some used residual sera obtained for other tests with virtually no risk factor data.

\section{Conclusion}

Results from the current systematic review show that in this low endemicity country, disease acquisition occurs in adulthood rather than childhood. The burden of disease is high; approximately 1 in 10 Canadians had been infected by ages 24-29. The increase in prevalence in young adults coincides with disease importation and increasing frequency of risk factors, most likely behavioral-related ones.

Gaps in seroprevalence data were also identified in this systematic review, rendering the application of current recommendations difficult. A nationwide prevalence survey for all Canadians is needed. This is essential to quantify the effectiveness of current recommendations [10] and conduct cost-effectiveness evaluations of alternative immunization programs, if necessary [69].

\section{Abbreviations}

G6 students grade 6 students

\section{Competing interests}

Funding for this systematic review was provided by GlaxoSmithKline Canada. BP and ACT are employed by GlaxoSmithKline. BD, GDS, VG, JO, and DS have received research funding from GlaxoSmithKline.

\section{Authors' contributions}

$\mathrm{BP}$ contributed to the development of the research question and methodology, project management, systematic review, data management and data analysis, derivation of charts and tables, and interpretation of the results. BD, GDS, DS contributed to the development of the research question and methodology, acquisition of unpublished data, and interpretation of the results. JO and VG contributed to the development of the research methodology, acquisition of unpublished data, derivation of charts and tables and interpretation of the results. ACT contributed to the development of the research methodology, project management, systematic review, derivation charts and tables and interpretation of the results. All of us contributed to the manuscript writing and approved the final version of the manuscript.

\section{Acknowledgements}

We would like to thank Perica Sever for her continued support. We would like to also thank Dr. Jun Wu for his guidance on how hepatitis $A$ cases were reported as a notifiable disease in Canada. Additionally, we would also like to thank Richard Foty for compiling the data and Yang Liu for her technical assistance.

\section{References}

I. Cuthbert JA: Hepatitis A: old and new. Clin Microbiol Rev 200I, I4:38-58.

2. Gust ID: Epidemiological patterns of hepatitis $A$ in different parts of the world. Vaccine 1992, 10 Suppl I:S56-S58.

3. Tapia-Conyer R, Santos JI, Cavalcanti AM, Urdaneta E, Rivera L, Manterola A, Potin M, Ruttiman R, Tanaka KJ: Hepatitis A in Latin America: a changing epidemiologic pattern. Am J Trop Med Hyg 1999, 61:825-829.

4. Bell BP, Shapiro CN, Alter MJ, Moyer LA, Judson FN, Mottram K, Fleenor M, Ryder PL, Margolis HS: The diverse patterns of hepatitis A epidemiology in the United States-implications for vaccination strategies. J Infect Dis 1998, I78:1579-I 584.

5. Notifiable disease annual summary 1997. Can Commun Dis Rep 1999, 25:119-121.

6. British Columbia Center for Disease Control: BC reportable communicable diseases, annual summary 1998. 1999:23-25.

7. Furesz J, Scheifele DW, Palkonyay L: Safety and effectiveness of the new inactivated hepatitis A virus vaccine. CMAJ 1995, I 52:343-348.

8. Scheifele DW, Ochnio J: Hepatitis A vaccine: is it being used to best advantage? CMAJ 2002, 167:44-45.

9. Practices ACl: Prevention of hepatitis $A$ through active or passive immunization. MMWR Morb Mort Wkly Rep 1999, 48: I-37.

10. Samandari T, Bell BP, Armstrong GL: Quantifying the impact of hepatitis A immunization in the United States, 1995-2001. Vaccine 2004, 22:4342-4350.

II. Immunization NAC: Canadian Immunization Guide 6th edition. Ottawa, Canadian Medical Association; 2002.

12. Wu J, Zou S, Giulivi A: Current hepatitis A status in Canada. Can J Infect Dis Med Microbiol 200I, I 2:34I-344.

13. Population and Public Health Branch HC: Notifiable disease incidence for years 1987-1 999. 2004.

14. Scheifele DW: Hepatitis A vaccines: the growing case for universal immunisation of children. Expert Opin Pharmacother 2005, 6:157-164.

15. Koff RS: Seroepidemiology of hepatitis A in the United States. J Infect Dis 1995, I7I Suppl I:S19-S23.

16. Armstrong GL, Bell BP: Hepatitis A virus infections in the United States: model-based estimates and implications for childhood immunization. Pediatrics 2002, 109:839-845.

17. elSaadany S, Gully P, Giulivi A: Hepatitis A, B, and C in Canada. Results from the National Sentinel Health Unit Surveillance System, 1993-1995. Can J Public Health 2002, 93:435-438. 
18. Payment P: Antibody Levels to Selected Enteric Viruses in a French-Canadian Population in the Province of Quebec. Immunol Infect Dis 1991, I:317-322.

19. Cook D, Wilton L, D P, Zou S, Sherman M, Krajden M: Prevalence of antibodies to hepatitis $A$ virus in a cohort of women of child-bearing age. 68th Canadian Association for Clinical Microbiology and Infectious Disease, Nov 5-8, 2000 - Ottawa 2000.

20. McAuley L, Pham B, Tugwell P, Moher D: Does the inclusion of grey literature influence estimates of intervention effectiveness reported in meta-analyses? Lancet 2000, 356:|228-|23|

21. Deeks Jj: Issues in the selection of a summary statistic for meta-analysis of clinical trials with binary outcomes. Stat Med 2002, 2 I: I575-1600.

22. Ochnio JJ, Scheifele DW, Fyfe M, Bigham M, Bowering D, Martiquet $\mathrm{P}$, Ho $\mathrm{M}$, Talling DN: The prevalence of hepatitis $\mathbf{A}$ in children in British Columbia. Can J Infect Dis Med Microbiol 2005, 16:175-179.

23. Muecke CJ, Beliveau C, Rahme E, Soto JC, Gyorkos TW: Hepatitis A seroprevalence and risk factors among day-care educators. Clin Invest Med 2004, 27:259-264.

24. Minuk GY, Zhang M, Wong SG, Uhanova J, Bernstein CN, Martin B, Dawood MR, Vardy L, Giulvi A: Viral hepatitis in a Canadian First Nations community. Can J Gastroenterol 2003, 17:593-596.

25. Ochnio JJ, Patrick D, Ho M, Talling DN, Dobson SR: Past infection with hepatitis A virus among Vancouver street youth, injection drug users and men who have sex with men: implications for vaccination programs. CMAJ 200I, I 65:293-297.

26. Smieja M, Cronin L, Levine M, Goldsmith CH, Yusuf S, Mahony JB: Previous exposure to Chlamydia pneumoniae, Helicobacter pylori and other infections in Canadian patients with ischemic heart disease. Can J Cardiol 200I, I 7:270-276.

27. Kiefer LA, Honish A, Predy G, Talbot JA: The seroprevalence of hepatitis $A$ and $B$ in people testing positive for hepatitis $C$. CMAJ 2000, I 62:207-208.

28. Allard R, Beauchemin J, Bedard L, Dion R, Tremblay M, Carsley J: Hepatitis A vaccination during an outbreak among gay men in Montreal, Canada, I995-1997. J Epidemiol Community Health 200I, 55:25I-256.

29. Moses S, Mestery K, Kaita KDE, Minuk GY: Viral hepatitis in a Canadian street-involved population. Can J Public Health 2002, 93:123-128

30. Roy E, Haley N, Leclerc P, Cedras L, Bedard L, Allard R: Seroprevalence and risk factors for hepatitis $A$ among Montreal street youth. Can J Public Health 2002, 93:52-53.

31. Ochnio Jl, Scheifele DW, Ho M: Hepatitis A virus infections in urban children--are preventive opportunities being missed? J Infect Dis 1997, 176:1610-1613.

32. De Serres G, Laliberte D: Hepatitis A among workers from waste water treatment plant during a small community outbreak. Occup Environ Med 1997, 54:60-62.

33. De Serres G, Levesque B, Higgins R, Major M, Laliberte D, Boulianne $N$, Duval B: Need for vaccination of sewer workers against leptospirosis and hepatitis A. Occup Environ Med 1995, 52:505-507.

34. Smieja M, Gnarpe J, Lonn E, Gnarpe H, Olsson G, Yi Q, Dzavik V, McQueen $M$, Yusuf $S$ : Multiple infections and subsequent cardiovascular events in the Heart Outcomes Prevention Evaluation (HOPE) Study. Circulation 2003, 1 07:25 I-257.

35. Embil JA, Manley K, White LA: Hepatitis A: a serological study in the Canadian Armed Forces. Mil Med 1989, I 54:46I-465.

36. Nicolle LE, Minuk GY, Postl B, Ling N, Madden DL, Hoofnagle JH: Cross-sectional seroepidemiologic study of the prevalence of cytomegalovirus and herpes simplex virus infection in a Canadian Inuit (Eskimo) community. Scand J Infect Dis 1986, I 8:19-23.

37. Minuk GY, Ling N, Postl B, Waggoner JG, Nicolle LE, Hoofnagle JH: The changing epidemiology of hepatitis $B$ virus infection in the Canadian north. Am J Epidemiol 1985, I 2 I:598-604.

38. Crewe MD, Embil JA, Garner JB: Prevalence of antibodies to hepatitis A virus in Nova Scotia children. Can Med Assoc J I 983, | 28: I 195-। | 97.

39. Minuk GY, Waggoner JG, Jernigan R, Nicolle LE, Postl B, Hoofnagle $\mathrm{H}$ : Prevalence of antibody to hepatitis A virus in a Canadian Inuit community. Can Med Assoc J 1982, 127:850-852.
40. Minuk GY, Nicolle LE, Postl B, Waggoner JG, Hoofnagle JH: Hepatitis virus infection in an isolated Canadian Inuit (Eskimo) population. J Med Virol 1982, 1 0:255-264.

4I. McFarlane ES, Embil JA, Manuel FR, Gorelick M: Prevalence of antibodies to hepatitis $A$ antigen in patients attending a clinic for treatment of sexually transmitted diseases. Sex Transm Dis 1980, 7:87-89.

42. Buchner BK, Staszak M, Duravetz J, Moore BP: Hepatitis markers detected in a Canadian blood donor population. Acta Haematol Pol 1980, I I:69-7I.

43. Minuk GY, Ding LX, Hannon C, Sekla $L$ : The risks of transmission of acute hepatitis $A$ and $B$ virus infection in an urban centre. J Hepatol 1994, 2 I: I 18-121.

44. Minuk GY, Uhanova J: Viral hepatitis in the Canadian Inuit and First Nations populations. Can J Gastroenterol 2003, I 7:707-7I 2.

45. McFarlane ES, Embil JA: Antibody to hepatitis A virus in young inmates. I Infect Dis 1982, I 46:442.

46. McFarlane ES, Embil JA: Prevalence of antibodies to Hepatitis A antigen in sera from patients with haematological malignancies. J Med Virol I98I, 8:49-53.

47. Wu H, Zaniewski G, Hong Z, Wu J, Dawood MR, Smart G, Geodhuis $N$, Giulivi A: Changes in the epidemiology of hepatitis A infection in Manitoba 1992-2003: Implications for vaccination strategies. Can J Infect Dis Med Microbiol 2004, I 5:356 (Abstract P25).

48. Duval B, De Serres G, Gilca V, Ochnio J, Scheifele DW: Nationwide Canadian study of hepatitis $A$ antibody prevalence among children eight to thirteen years old. Pediatr Infect Dis J 2005 24:514-519.

49. Ochnio JJ, Scheifele DW, Marion SA, Bigham M, Ho M: Hepatitis A virus (HAV) infections in young adults in British Columbia: feasibility of a novel mail-based approach to population sampling. Proceedings of the 7th Annual Conference on Vaccine Research Arlington, VA, USA 2004, Abstract P4:

50. Harb J: Hepatitis A in the Northern Interior of British Columbia: An Outbreak Among Members of a First Nations Community. Can Commun Dis Rep 2000, 26: I57-I6I.

51. Levy I, Chen D, Sherman M, Smith D, Krajden M: Hepatitis A virus seroprevalence in 1,000 university students in Toronto. Can Commun Dis Rep 200I, 27:93-96.

52. EL FJ, I K, M C, M P, R N, N D, R G: Seroprevalence of hepatitis A (HAV), hepatitis B (HBV), human parvovirus B I 9 (HPV $B$ I9) and toxoplasmosis (Toxo) in Toronto infant-toddler day care providers. 35th Interscience Conference on Antimicrobial Agents and Chemotherapy, Sept 17-20, 1995 1995, San Francisco, CA. Washington: American Society for Microbiology, 1995 (Abstract KI54):

53. Ochnio J]: Traveler Seroprevalence Data in Vancouver. 1995

54. Kocuipchyk FR: Seroprevalence of hepatitis $\mathbf{A}$ antibodies in travellers at the Edmonton Travellers' Health Clinic-. Alberta. Can Commun Dis Rep 1995, 21 :65-71.

55. Richer G, Chen YY, Huet PM: Incidence of hepatitis non-A, nonB compared with types $A$ and $B$ in hospital patients. Can Med Assoc J 1982, I 27:384-386.

56. Basrur V: Toronto Staff Report: Hepatitis A at a Toronto grocery store. Toronto Public Health 2002

57. Pollett GL: Middlesex-London Health Unit Report No. I 28-02: Hepatitis A in a foodhandler. 2002.

58. Authority VCH: Hepatitis $\mathbf{A}$ in a Capers grocery store in Vancouver. Infectious Diseases News Brief 2002

59. Jacobsen $\mathrm{KH}$, Koopman JS: Declining hepatitis A seroprevalence: a global review and analysis. Epidemiol Infect 2004 I32:1005-1022

60. Beutels $M$, Van Damme $P$, Vranckx $R$, Meheus A: The shift in prevalence of hepatitis $\mathbf{A}$ immunity in Flanders, Belgium. Acta Gastroenterol Belg 1998, 6 I:4-7.

61. Morris-Cunnington MC, Edmunds WJ, Miller E, Brown DW: A population-based seroprevalence study of hepatitis $A$ virus using oral fluid in England and Wales. Am J Epidemiol 2004 I 59:786-794.

62. Prodinger WM, Larcher C, Solder BM, Geissler D, Dierich MP: Hepatitis $A$ in Western Austria--the epidemiological situation before the introduction of active immunisation. Infection 1994 , 22:53-55. 
63. Bottiger $M$, Christenson B, Grillner L: Hepatitis A immunity in the Swedish population. A study of the prevalence of markers in the Swedish population. Scand J Infect Dis 1997, 29:99-102.

64. Global impact of hepatitis A virus infection: changing patterns. In Viral Hepatitis and Liver Disease Edited by: Hollinger FB LSMMHS. Baltimore, Williams and Wilkins; 2005: 14-20.

65. Hadler SC, cala de MM, Bensabath G, Martinez DM, Schatz G, Fields $\mathrm{HA}$ : Epidemiology of hepatitis delta virus infection in less developed countries. Prog Clin Biol Res 199I, 364:2I-3I.

66. Hutin YJ, Pool V, Cramer EH, Nainan OV, Weth J, Williams IT, Goldstein ST, Gensheimer KF, Bell BP, Shapiro CN, Alter MJ, Margolis HS: A multistate, foodborne outbreak of hepatitis $A$. National Hepatitis A Investigation Team. N Engl J Med 1999, 340:595-602.

67. Rosenblum LS, Mirkin IR, Allen DT, Safford S, Hadler SC: A multifocal outbreak of hepatitis A traced to commercially distributed lettuce. Am J Public Health 1990, 80:1075-1079.

68. Fulford M, Keystone JS: Health Risks Associated with Visiting Friends and Relatives in Developing Countries. Curr Infect Dis Rep 2005, 7:48-53.

69. Krahn M, Remis R, Hogg R, Weber A, Wu J, Sherman M, Shimian Z: Universal hepatitis $A$ vaccination for adolescents and children in Canada: a cost effectiveness analysis. 2005.

\section{Pre-publication history}

The pre-publication history for this paper can be accessed here:

http://www.biomedcentral.com/1471-2334/5/56/prepub

\section{Publish with Biomed Central and every scientist can read your work free of charge}

"BioMed Central will be the most significant development for disseminating the results of biomedical research in our lifetime. "

Sir Paul Nurse, Cancer Research UK

Your research papers will be:

- available free of charge to the entire biomedical community

- peer reviewed and published immediately upon acceptance

- cited in PubMed and archived on PubMed Central

- yours - you keep the copyright

Submit your manuscript here:

http://www.biomedcentral.com/info/publishing_adv.asp 